$\therefore N F-88115 \%-60$

CONF-881151--60

DE89 009870

\title{
STATUS OF THE POSITIVE ION INJECTOR FOR ATLAS ${ }^{\star}$
}

APR $1 \leq 9989$

P. K. Den Hartog, R. Benaroya, J. M. Bogaty, L. M. Bollinger, B. E. Clifft,

S. L. Craig, D. Henderson, P. Markovich, F. Munson, J. M. Nixon,

R. C. Pardo, D. Phillips, K. W. Shepard, I. Tilbrook, and G. Zinkann

Physics Division, Argonne National Laboratory Argonne, IL 60439-4843 USA

Abstract

The positive ion injector project will replace a High Voltage Engineering Corp. model FN 9 MV tandem electrostatic accelerator as the injector into the ATLAS superconducting heavy ion linear accelerator. It consists of an electron cyclotron resonance (ECR) ion source on a $350-\mathrm{kV}$ platform injecting into a linac of individually phased superconducting resonators which have been optimized for ions with velocities as low as $B=0.009$. The resulting combination will extend the useful mass range of ATLAS to projectiles as heavy as uranium, while increasing the beam currents available by a factor of 100 .

"Work supported by the U. S. Department of Energy, Nuclear Physics Division, under Contract W-31-109-Eng-38

\section{DISCLAIMER}

This report was prepared as an account of work sponsored by an agency of the United States Government. Neither the United States Government nor any agency thereof, nor any of their employees, makes any warranty, express or implied, or assumes any legal liability or responsibility for the accuracy, completeness, or usefulness of any information, apparatus, product, or process disclosed, or represents that its use would not infringe privately owned rights. Reference herein to any specific commercial product, process, or service by trade name, trademark, manufacturer, or otherwise does not necessarily constitute or imply its endorsement, recommendation, of favoring by the United States Government or any agency thereof. The views and opinions of authors expressed herein do not necessarily state or reflect those of the United States Government or any agency thereof. 


\section{Introduction}

The Argonne Tandem-Linac Accelerator System (ATLAS) has been in use since 1978 and has logged over 35,000 hours of beam time for research, providing ions of masses up to $A=127$. The tandem accelerator is limited as an injector, however, by the poor performance of negative ion sources for some ion species, the deleterious effect on beam quality of stripping, the short lifetime of stripping foils, and the low transmission for heavy ions.

A new positive ion injector (PII) now under construction will address these shortcomings. The design has been discussed extensively elsewhere..$^{1,2}$ Briefly, it consists of an electron cyclotron resonance (ECR) ion source on a $350 \mathrm{kV}$ platform injecting into a linac optimized for very slow $(\beta<009 \mathrm{c})$ ions. Beam optics calculations have shown that the radial defocusing introduced by the acceleration process can be minimized by the use of extremely short but high gradient superconducting resonators alternated with short focal length superconducting solenoid magnets. ${ }^{3}$

The project is being constructed in three phases. Phase 1 , now under construction, will develop the technology and produce a 3-MV injector. In conjunction with ATLAS, it will provide energies nearly equal to those achieved with the existing tandem accelerator for ions up to mass $A=70$ and beam intensities up to 100 times greater. For $70<A<120$, the acceleration performance of the Phase 1 linac will exceed the capabilities of the tandem injector. Phases 2 and 3, scheduled for completion in early 1990 and 1991, respectively, will add 2 additional cryostats of 6 resonators each to bring the total accelerating voltage of the injector to $12 \mathrm{MV}$. With the completion of the PII injector, the ATLAS accelerator will be capable of providing beam energies of up to $20 \mathrm{MeV} / \mathrm{A}$ for the lightest ions and 7-10 MeV/A for uranium. Figure 1 is a layout of the entire ATLAS facility showing the location and scale of the PII upgrade and the existing accelerator and experimental areas.

\section{Resonator development}

Four new classes of superconducting resonators ${ }^{4}$ were designed for the accelerator. All are quarter wave interdigital 4-gap structures of niobium with explosively bonded niobium-copper housings. Figure 2 shows the layout of the PII and an exploded view of the resonator configuration for Phase 1. Five 
resonators will be used including at least one resonator of each class. All of the resonators have been fabricated and test results are summarized in Table 1. The initial minimum performance projection of $3 \mathrm{MV} / \mathrm{m}$ has been substantially exceeded in each class.

The ancillary components of the PII resonators will be similar in concept to those currently in use on the ATLAS split-ring resonators, but many improvements have been made. The fast-tuner system, resonator control modules, and reference frequency distribution system, have all been redesigned. The upgrades were driven by a desire to increase the performance of the existing linac by incorporating new technology and by the lessons of ten years of superconducting linac operating experience.

The redesign of the phase stabilization system has been particularly beneficia!. Microphonic fluctuations in the RF eigenfrequency of superconducting slow-wave structures must be compensated, for many applications, by a fast-tuning system in order to control the RF phase. The tuning system must handle a reactive power, $P=2 d \omega U$, where $d \omega$ is the tuning range and $U$ the $R F$ energy content of the resonant cavity. The accelerating field of many of the superconducting cavities forming the ATLAS superconducting linac has been limited by the RF power capacity of the presently used fast tuner. A new system has been developed utilizing PIN diodes operating immersed in liquid nitrogen, with the diodes being controlled by a high-voltage VMOS FET driver. The new system has operated at reactive power levels above $20 \mathrm{KVA}$, a factor of four increase over the earlier design. This will enable the PII resonators to be reliably phase locked at higher accelerating fields than the existing ATLAS resonators. We intend to begin retrofitting the ATLAS accelerator to gain these same benefits later this year.

Slow frequency shifts of larger amplitude are handled in a different manner. The method employed on the split-ring resonators has worked well, but the vastly different geometry and increased rigidity of the interdigital resonators required a redesign of the mechanical components. The slow-tuner system for the interdigital resonators has been designed and tested and the frequency shifts obtained were sufficient for online operation.

A fully dressed test of a $\beta=0.025$ interdigitial resonator with the newly designed fast tuner, new drive electronics, and slow tuner, has been successfully operated at $4.25 \mathrm{MV} / \mathrm{m}$ with 4.5 watts of refrigeration at $4.2 \mathrm{~K}$. 


\section{Timing}

A primary requirement of the PII is that it reproduce or improve upon the excellent beam quality available from a tandem electrostatic accelerator. Since the emittance growth within the linac is largely determined by the quality of the injected beam, it is important that the bunching and beam transport not degrade the transverse emittance and energy spread from the ECR source.

To achieve this goal, beam bunching for the injector is done in two stages, with the first buncher located on the ECR high-voltage platform, and the second buncher placed immediately in front of the first resonator cryostat. The first buncher is a gridded, harmonic, 12- $\mathrm{MHz}$ buncher, identical to the existing pre-tandem buncher, but with four harmonics rather than three, to optimize the waveform linearity. It forms a virtual image for the second-stage buncher which will be a room temperature spiral-loaded 2-gap structure. The second-stage buncher has been constructed and tested, producing the required effective accelerating potential of $49 \mathrm{kV}$ with $400 \mathrm{w}$ of input power. The resonator operated at $65 \mathrm{kV}$ in a pulsed mode without discharges. A parallel plate chopper is located on the beamline upstream from the second-stage buncher to remove tails.

The voltage of the ECR platform must be stabilized to $\Delta \mathrm{V} / \mathrm{V}<1 \times 10^{-4}$ to avoid adding significant energy spread to beam. Measurements of the platform stability have shown a ripple of $<5$ volts peak to peak at $300 \mathrm{kV}$, mostly from capacitive coupling through the isolation transformers, but well within this limit. If necessary, a feedback system that has been used on the existing tandem negative ion source platform can be used to reduce the ripple even further.

Variation in path length of ions as they traverse the dipole magnets could also degrade the beam pulse timing. The use of an isochronous beam transport system will prevent this from occurring. Calculations predict that second order effects will not be significant.

\section{Timing diagnostics}

Beam pulse timing diagnostics are required at the linac entrance, since the longitudinal and transverse emittance growth of the beam as it passes through tine linac is sensitive to the shape of the longitudinal phase ellipse of the injected beam . 
Silicon surface barrier detectors have been used for beam pulse timing diagnostics measurements at ATLAS, but for the PII, beam energies will be too low to achieve the required resolution. In addition, a nondestructive detector that can be used continuously during accelerator operation is desirable. A microchannel plate detector system is under development. The detector consists of a thin $(10 \mu \mathrm{m})$ wire axially mounted in a cylindrical electrostatic field. The ion beam enters and exits through $8.5 \mathrm{~mm}$ apertures orthogonal to the wire. Electrons generated at the wire surface by ion collisions are swept along radial lines, and those which exit at nearly 90 degrees to the beam pass through a small aperture into the microchannel plate. Calculations have shown that the path length differences of the electrons should be small, thus the inherent resolution of the detector should be limited only by the microchannel plate. We hope to eventually realize a detector resolution of $\approx 60 \mathrm{ps}$; and initial off-line test results have been encouraging. The tests were performed using a $241 \mathrm{Am}$, 5.5-MeV alpha source to emulate the accelerator beam. The microchannel plate signal was measured relative to the alpha particle induced signal of a silicon detector. Timing resolutions of $300 \mathrm{ps}$ have been achieved, but the measurements have been hampered by low counting rates and by the energy degradation of the alpha particles passing through the wire. In beamline use, the microchannel plate signal will be measured relative to an RF reference signal. A test with an actual accelerator beam is expected in mid-November 1988.

\section{Mass resolution}

The mass resolution of the PII injector is provided by two successive 90 degree magnets. The first magnet is on the ECR platform, has a mass resolution of $\mathrm{m} / \Delta \mathrm{m} \approx 100$, and produces an image at the horizontal slit which forms the object of the second 90-degree magnet. The second magnet produces its image at a slit located at the center of the isochronous 180-degree bend and has a resolution of $\mathrm{m} / \Delta \mathrm{m} \approx 400$. Because the ECR source will always produce many unwanted ions, some with identical magnetic rigidity, some coritamination will be unavoidable for certain isotope and charge-state combinations. In these cases, an adjacent charge state may need to be selected with some loss of intensity or energy. The use of a velocity selecting chopper to remove beam pulse tails will also contribute to the overall mass resolution of the beamline, since the time resolution of the chopper is a function 
of the vertical phase space, while the magnetic system resolution is a function of the horizontal phase space.

\section{ECR source}

The Electron Cyclotron Resonance source 5 has operated for over a year, providing beams for atomic research on a temporary beamline. The source has produced beam currents of gaseous materials comparable with other ECR sources of similar design and development is proceeding on methods of ionizing solid materials. Experiments have been performed using wires of nickel and copper fed radially into the ECR region, and most recently, an oven was used to introduce cesium vapor into the source. The performance of the source and a discussion of the high-voltage platform are reported in a separate paper at this conference. 6

\section{Control system}

The computer control system that will be used for the PII accelerator will be based on the technology developed for the ATLAS linac. The system uses Digital Equipment Corp. PDP 11 computers linked together through a DECNET ${ }^{\mathrm{TM}}$ local area network and they communicate with the accelerator through a CAMAC byte-seriai highway. A separate control console for the ECR and PII linac allows development work to proceed without interfering with the ongoing operations of the ATLAS accelerator. Minor changes have been made in the operator interface to utilize current display technology and to improve the ease of use.

In the past all control software was custom designed and locally written, but inexpensive and powerful software for personal computers has become increasingly available. We have decided to use one of these products ${ }^{7}$ in the control of the ECR source and the cryogenics monitoring. The effectiveness of this package will be evaluated to determine if a distributed control system utilizing commercial software will be practical in other areas.

\section{Cryogenics}

To accommodate the PII injector an additional CTI 2800 refrigerator will be installed, adding 200 watts of additional capacity, and the original CTI 1400 machine will be decommissioned. This will bring the total refrigeration capacity 
of the system to 700 watts. The distribution line will be installed during October of 1988 and the refrigerator will be installed in the spring of 1989.

\section{Conclusion}

Phase 1 of the PII project is proceeding on schedule and many of the significant construction milestones have been passed. The first cryostat is in place and has been connected to the liquid ile distribution system, the resonators and solenoids will be installed in November of 1988, and the entire assembled cryostat will be cooled later that month.

The beamline connecting the ECR source and the injector is nearly complete. By late November, beam transmission and bunching tests will commence; culminating in beam accelerated by the linac by late 1988 or early 1989. Following a period of performance tests, it is planned to begin using the PII in conjunction with ATLAS for experiments in February 1989. Initially, operations of the PII will be alternated with operations of the tandem injector, allowing development to proceed and operating experience to be acquired. Interruptions will continue to occur until after the third cryostat is installed for Phase 3 , but the continued use of the tandem injector during these periods will allow ATLAS beams to remain available. 


\section{References}

(1) ATLAS Uranium Upgrade Project, Argonne Physics Division report, (Aug 1986).

(2) R. C. Pardo, L.M. Bollinger and K. W. Shepard, Nucl. Inst. and Meth., B24/24(1987)746-751.

(3) R. C. Pardo, K. W. Shepard, M. Karls, Proceedings of the 1987 Particle Accelerator Conf.,IEEE catalog no. 87CH2387-9, Washington D.C. , March 16-19, 1987 , pp. 12281230 .

(4) K. W. Shepard, Proceedings of the 1987 Particle Accelerator Conf.,IEEE catalog no. 87CH2387-9, Washington D.C. , March 16-19, 1987 , pp. 1812-1814.

(5) R. C. Pardo, P. J. Billquist, and J. E. Dey, Proceedings of the Int. Conf. Phys. Multicharged lons and Intl. Wkshp. E.C.R. Ion Sources, Sept. 12-16, 1988, to be published in Le Journal de Physique.

(6) R. C. Pardo and P. J. Billquist, 10th Int'l. Conf. on the Appl. of Accel. In Research and Industry, Denton, TX, Nov. 7-9, 1988.

(7) The Fix ${ }^{\mathrm{TM}}$, Intellution Corp., 35 Perwal St., Westwood, MA 02090. 
Table 1

PII Prototype Resonator Performance at $4.2 \mathrm{~K}$.

$\begin{array}{lcccc}\begin{array}{l}\text { Resonator } \\ \text { Class }\end{array} & \begin{array}{c}\text { freq } \\ (\mathrm{Mhz})\end{array} & \beta & \begin{array}{c}\mathrm{E}_{\mathrm{a}(\mathrm{max})} \\ (\mathrm{MV} / \mathrm{m})\end{array} & \begin{array}{c}\mathrm{E}_{\mathrm{a}}(4 \text { watts }) \\ (\mathrm{MV} / \mathrm{m})\end{array} \\ 11 & 48.5 & .009 & 10.1 & 6.5 \\ 12 & 48.5 & .016 & 5.8 & 4.6 \\ 13 & 48.5 & .025 & 6.0 & 4.1 \\ 14 & 72.75 & .037 & 5.3 & 4.1\end{array}$




\section{Figure Captions}

Figure 1. Layout of entire ATLAS facility showing the location of the Positive Ion Injector on the lest side of the figure.

Figure 2. Detailed layout of the Positive Ion Injector showing the configuration of resonators and solenoids that will be used in Phase 1 of the project. 


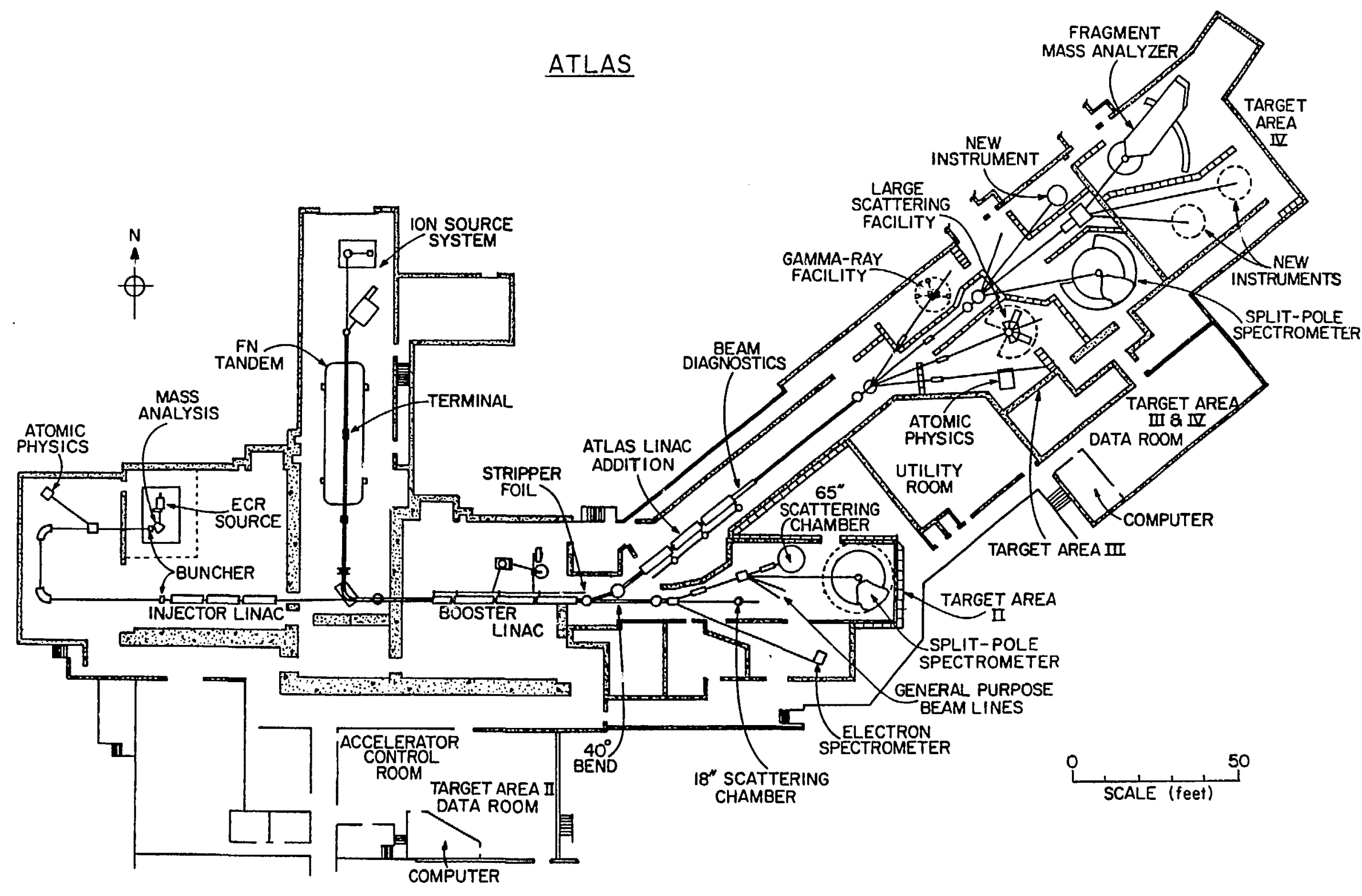


ANL-P-18,482

ARGONNE POSITIVE ION INJECTOR SYSTEM

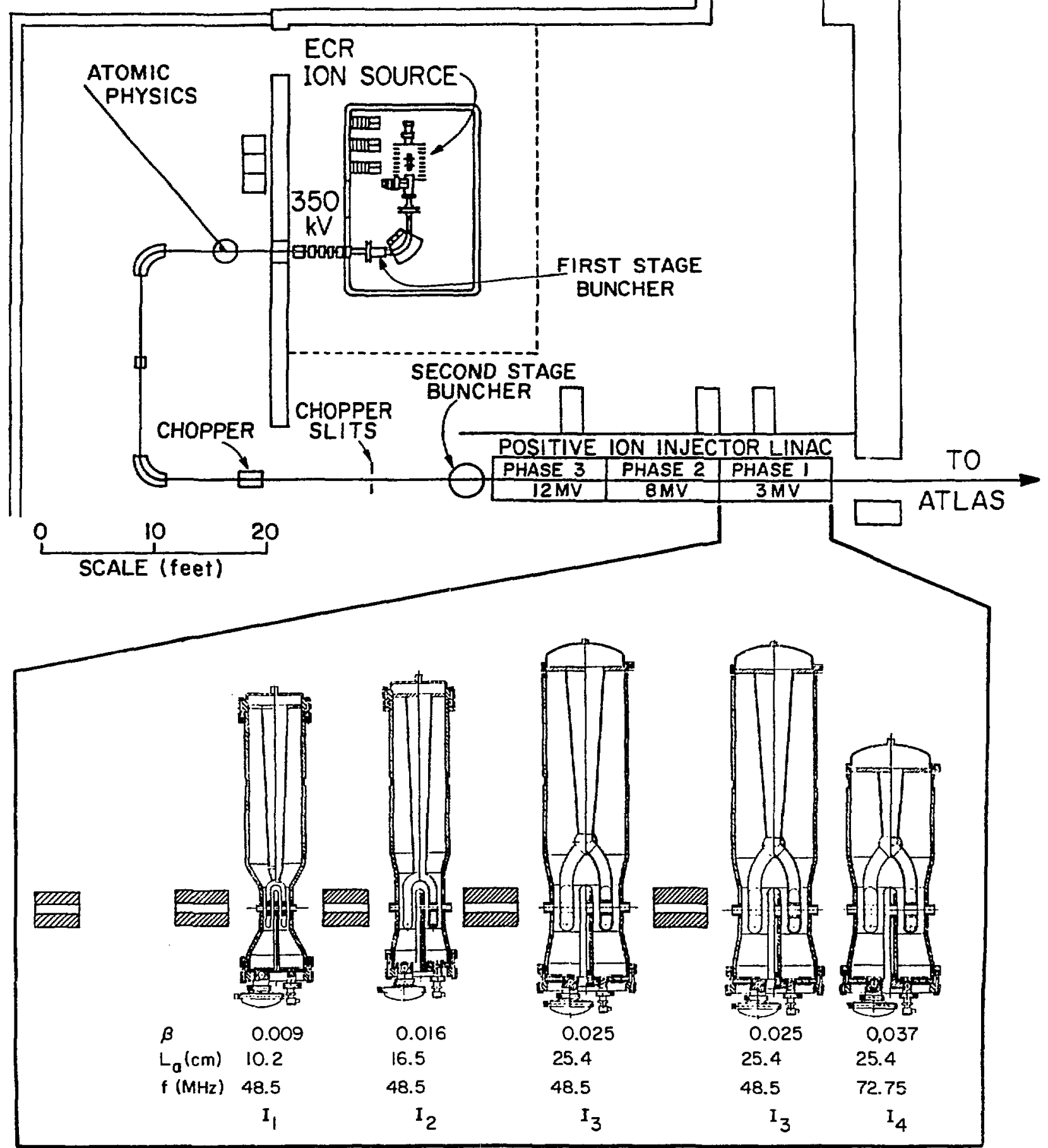

Fig. 2 